Article

\title{
A Velocity-Aware Handover Trigger in Two-Tier Heterogeneous Networks
}

\author{
Asmae Ait Mansour ${ }^{1, *(\mathbb{B})}$, Nourddine Enneya ${ }^{2}$ and Mohamed Ouadou ${ }^{1}$ \\ 1 LRIT-CNRST (URAC N 29), Faculty of Sciences, Mohammed V University, Rabat 1014, Morocco; \\ ouadou55@gmail.com \\ 2 Laboratory of Informatics, Systems and Optimization, Faculty of Sciences, Ibn Tofail University, Kenitra 133, \\ Morocco; enneya@gmail.com \\ * Correspondence: asmae.ait@gmail.com; Tel.: +212-619-682-371
}

Received: 27 November 2017; Accepted: 11 January 2018; Published: 15 January 2018

\begin{abstract}
The unexpected change in user equipment (UE) velocity is recognized as the primary explanation for poor handover quality. In order to resolve this issue, while limiting ping-pong (PP) events we carefully and dynamically optimized handover parameters for each UE unit according to its velocity and the coverage area of the access point (AP). In order to recognize any variations in velocity, we applied Allan variance (AVAR) to the received signal strength (RSS) from the serving AP. To assess our approach, it was essential to configure a heterogeneous network context (LTE-WiFi) and interconnect Media-Independent Handover (MIH) and Proxy Mobile IPv6 (PMIPv6) for seamless handover. Reproduction demonstrated that our approach does not only result in a gain in relatively accurate velocity but in addition reduces the number of PP and handover failures (HOFs).
\end{abstract}

Keywords: mobility management; heterogeneous networks (HetNets); IEEE $802.21 \mathrm{MIH}$; Proxy MIPv6; Network Simulator 3 (NS3)

\section{Introduction}

For new-generation wireless systems, researchers aspire to a future with better communication infrastructure with respect to quantity and quality. A fundamental area of application for heterogeneous systems is in different real-time services requiring a high data rate in different mobility conditions with a short delay. To accomplish convergence, heterogeneous technologies are incorporated over an Internet Protocol-based (IP-based) core. Currently, Internet Protocol Version 4 (IPv4)-based centers are set up to accomplish convergence. The Internet Engineering Task Force has comprehended the disadvantages of the IPv4-based network and has proposed a switch to IPv6. The change to IPv6 cores is necessary in order to accomplish perfect convergence [1] and to assist unique Internet addresses because of the increased number of cellphones.

These days, clients may utilize fast vehicular systems such as bullet trains. There thus exists significant enthusiasm for velocity following because of the link between velocity and handover failures (HOFs). This is because if velocity reaches a point where the time across the handover zone does not match the base handover delay, the handover procedure cannot be finished and calls will drop. On the other hand, recurrent handover is caused by the high-velocity mobility of mobile terminals [2]. The wireless access issues in rapid high-velocity mobility situations are as follows [3]: (1) Frequent handover, which generates frequent cell re-selection, communication quality degradation, and data service unavailability; (2) Doppler recurrence movement and fast fading, which causes an excruciatingly low correspondence connection rate, difficulties in handover initiation, and various handover failures; and (3) Large car body loss and multipath loss, which causes feeble intra-train signals. Usually, frequent handover brings the issue of packet loss and packet reordering. 
The consumer demands rapid data access with consistent connectivity to the best-connected network at any time, anywhere. In this way, a principal problem in high-velocity heterogeneous wireless systems is how to accomplish quick handover. Knowledge of the velocity of the user equipment (UE) represents a basic part of handover execution. Moreover, the inter-working of various wireless access technologies aims to fulfill the UE with respect to quality of service (QoS). These difficulties spur the need to utilize Media-Independent Handover (MIH). A definitive motivation behind IEEE $802.21 \mathrm{MIH}$ is to provide a mobility process unattached to media by offering helpful data about the link layer and candidate networks [4]. This urges us to embrace a standout method amongst the most illustrative endeavors for mobility management. Proxy Mobile IPv6 (PMIPv6) can be used to defeat the host-based issues by supporting mobility management without host inclusion in mobile signaling.

This paper will present consistent handover execution in view of the coordination between PMIPv6 and MIH, in light of the fact that guaranteeing consistent inter-networking of a heterogeneous network requires the flawless participation of all the layers. The rest of this paper is presented as follows: Section 2 introduces other approaches that estimate velocity and support handover performance for mobile UE. Section 3 depicts velocity observation utilizing Allan variance (AVAR) and introduces the proposed mobility management flow process. Section 4 gives a simulation configuration, scenario, and results. Discussion and concluding remarks are given in Sections 5 and 6, respectively.

\section{Related Work}

The settlement of where and when to perform handover is a driving issue in the realm of mobile communication. It is a pivotal measure for the handover process in order to grant universal handover, accomplish the best QoS in a heterogeneous environment, and provide the best Quality of Experience (QoE) for mobile UE. This decision requires us to investigate UE velocity and assemble data regarding the available services of the competitor networks in order examine this information with a specific end goal to settle on a decision at a suitable time.

\subsection{Velocity and Handover Performance}

In wireless communication networks, the estimation of the mobile users' velocity is an essential part of upgrading network execution. Subsequently, in recent years portable velocity estimation has been broadly considered in the literature [5-8]. Handover count-based strategy [6] is firmly related to the velocity estimation approach, where the UE's mobility is evaluated utilizing the handover quantity achieved by the UE in a predefined time window. Existing Long-Term Evolution (LTE) and LTE-Advanced innovations utilize the handover-count strategy to classify the mobility condition of UE into three large classes: low, medium, and high mobility. Hypothetically, the sojourn time-based velocity estimation in [7] resulted more exact than handover count-based velocity estimation. The author appraised the UE velocity in light of sojourn time samples and small cell base station (SBS) density. However, this work used an unrealistic mobility system. Furthermore, indeterminate estimation in the limit of small cells was experienced. In [5], the radial velocity was gained through the most extreme Doppler spread estimation of the received signal in mobile communications, although it required an extensive perception interim and signal noise ratio (SNR) higher than $30 \mathrm{~dB}$.

Tracking velocity can fundamentally enhance handover algorithm execution by simultaneously decreasing both the handover quantity and the handover delay, as demonstrated in $[9,10]$. The authors of [9] perceived the effect of the handover execution on communication quality, and they thus proposed a basic, effective, aware handover management scheme endeavoring to maintain longer service continuation with serving base stations (BSs) and decrease the handover rate and its related signaling. Using mathematical expressions, they demonstrated that the proposed handover method accomplished an impressive gain in terms of throughput. However, they supposed during handover execution that no information was transmitted. Taking into account that their numerical results for delay achieved 
high esteem, allowing communication and measuring packet loss through handover is attractive and will help produce the QoS achieved by their system. The authors of [10] demonstrated how low quality in handover execution could result in a severe interruption and call drops in communication, which remains a pressing issue yet to be solved. The authors thus examined a handover system in view of distance data for LTE high-velocity rail networks. The strategy for choosing a handover reference point depended on the situation given specific channel conditions. The outcomes demonstrated that a small region appropriate to triggering in the overlap area can be obtained, and handover execution can be expanded by diminishing the HOF probability and wiping out ping-pong (PP) handover.

\subsection{Handover Triggering}

To the best of the authors' knowledge, there are no investigations in the literature that survey the performance limits of the sudden change of velocity in handover performance, since the majority velocity-based handover improvement approaches concentrate on velocity estimation and not its variety. In the work of Yang et al. [11], the authors began to evaluate velocity after an impressive number of HOFs occurred. The authors calculated velocity in view of the received signal strengths (RSSs) of two diverse BSs, with which the handover margin (HM) was balanced accordingly. The numerical outcomes demonstrate that handover triggering velocity-based methods can essentially decrease the call drop rate, particularly when the velocity is high, which contributes to improved handover performance.

In parallel, there is a diverse handover trigger idea which depends on criteria other than the velocity, for example, RSS-based activating systems. This idea was utilized by [12] for a log-normal fading environment, where the handover algorithm was examined based on the level crossing of the difference between the RSSs. A strategy based on an improved velocity handover triggering system was proposed in [13]. The proposed scheme effectively limits the system load to avoid link crash. In addition, the introduced scheme augments the resource usage. Consequently, UE can utilize the system to maximum capacity. This triggering technique additionally decreases handover latency and the handover dropping rate. Various handover triggering schemes are location-based, such as in [14] where a handover management system for LTE femtocells was discussed. They used a modified self-organizing map (SOM) to learn the locations of the indoor environment. The triggering technique can also benefit from a prediction model, as proposed in [15].

Furthermore, in [16] the authors investigated multiple guidelines like signal strength and available bandwidth in the selection of the optimal target cell, which could increase the success rate and thereby reduce blocking. For enhancing the quality of the video and multimedia traffic, the work in [17] was found. The authors of this work proposed techniques for triggering handover based on the quality of multimedia information. However, different issues exist, such as estimating the correct amount of data for handover triggering.

\section{Overall Handover Procedures}

\subsection{Allan Variance and RSS}

The Allan change was presented 50 years ago for investigating the security of frequency standards. Due to its metrological characteristics, it has been utilized as part of accelerometry, geophysics, geodesy, astrophysics, and even finance. For example, AVAR can segregate distinctive sorts of noise described by various power laws into spectral densities. Along these lines, the principal application of AVAR for time series analysis is in deciding the signal scatter level, and in spectral analysis it primarily aims to recognize the predominant noise type in a time series.

Noise assessment in physical estimation time series is a critical measure of statistical attributes and overall quality. Among the best approaches to deal with investigating noise estimation (scatter) we have AVAR, which was initially introduced to measure how much the frequency of an oscillator changes from one period $\tau$ to the next. Since then, AVAR has become an intense measurable technique 
for time series analysis, especially for the examination of geodetic and astronomical observations. To date, it has only been used for time arrangement investigation. Accordingly, in our paper we will apply AVAR to RSS to recognize any change in velocity. As shown by [18] AVAR has the capacity to recognize colored noise related to RSS and can assist in the identification and characterization of these components. The AVAR is a fluctuation for two-sample $N_{m}=2$, where $X(t)$ is the signal to be examined. Utilizing the IEEE-prescribed definition of frequency in view of the endpoint phases [19], the AVAR at various times $t_{m}$ can be written as:

$$
\sigma_{\tau}^{2}=\frac{1}{2} E\left\{\left(\bar{X}\left(t_{m}+\tau\right)-\bar{X}\left(t_{m}\right)\right)^{2}\right\}=\frac{1}{2} E\left\{\left(\bar{X}_{m+1}-\bar{X}_{m}\right)^{2}\right\}
$$

where

$$
\bar{X}_{m}=\frac{1}{\tau} \int_{t_{m}}^{t_{m}+\tau} X(t) d t
$$

and $\bar{X}_{m+1}$ and $\bar{X}_{m}$ are two successive integrations over a duration $\tau$. Since the RSSs are considered observations points succeeding one another in time, this equation will be represented in the discrete case taking $\left(X_{j}\right)_{j=1, . . N}$ as a stochastic process whose realizations $X_{j}$ are made at constant time intervals $\tau_{0}$ (see Figure $1[20]$ ) and $m=\frac{\tau}{\tau_{0}}$.

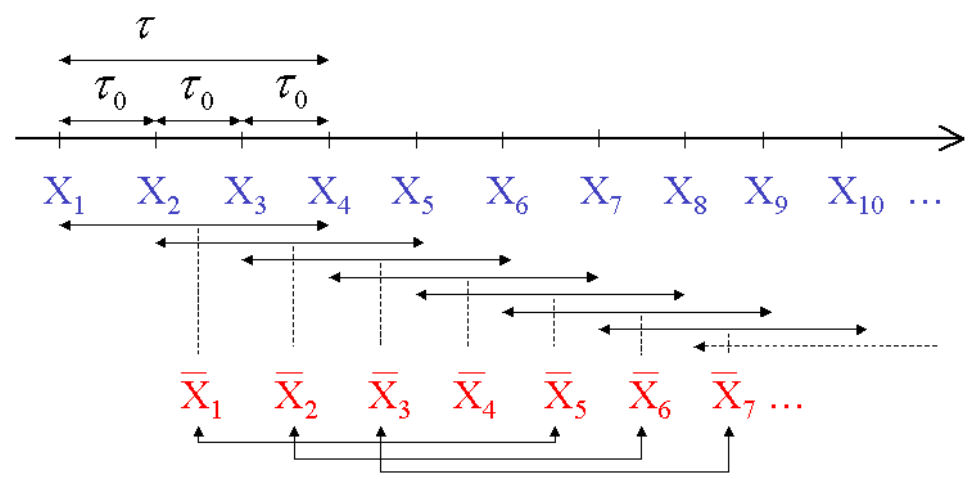

Figure 1. Principle of sub-sampling by Allan variance.

If we develop the empirical mean which is in fact an estimator of the expectation, the estimate of the variance of Allan used in this work is:

$$
\sigma^{2}(\tau)=\frac{1}{2 *\left(N-2\left(\frac{\tau}{\tau_{0}}\right)\right)} \sum^{N-2\left(\frac{\tau}{\tau_{0}}\right)}\left(\bar{X}_{k+\frac{\tau}{\tau_{0}}, \frac{\tau}{\tau_{0}}}-\bar{X}_{k, \frac{\tau}{\tau_{0}}}\right)^{2}
$$

Our study is based on the standard wireless RSS signal model given by:

$$
P_{y}(t)=P L_{0}-10 \gamma \log \left(\frac{d}{d_{0}}\right)+X_{g}
$$

where $P L_{0}$ is the path loss at the reference distance $d_{0}, \gamma$ is the path loss exponent, and $X_{g}$ is a normal (or Gaussian) random variable with zero mean reflecting the attenuation (in decibels) caused by flat fading. In our case we suppose that there is no fading, and therefore the distance is the only term varying with time.

As presented in Figures 2 and 3 the results of the AVAR of the RSS sample can allow the detection of a change in velocity. In order to detect this variation, it was decided to calculate the angle $\Theta_{t}$ between the two tangent lines for two adjacent intervals. The decision is target-based with $\Delta \Theta=\Theta_{t_{1}}-\Theta_{t_{2}} \geq 1 \%$, where $\Theta_{t_{1}}$ and $\Theta_{t_{2}}$ are two successive values of $\Theta_{t}$. 

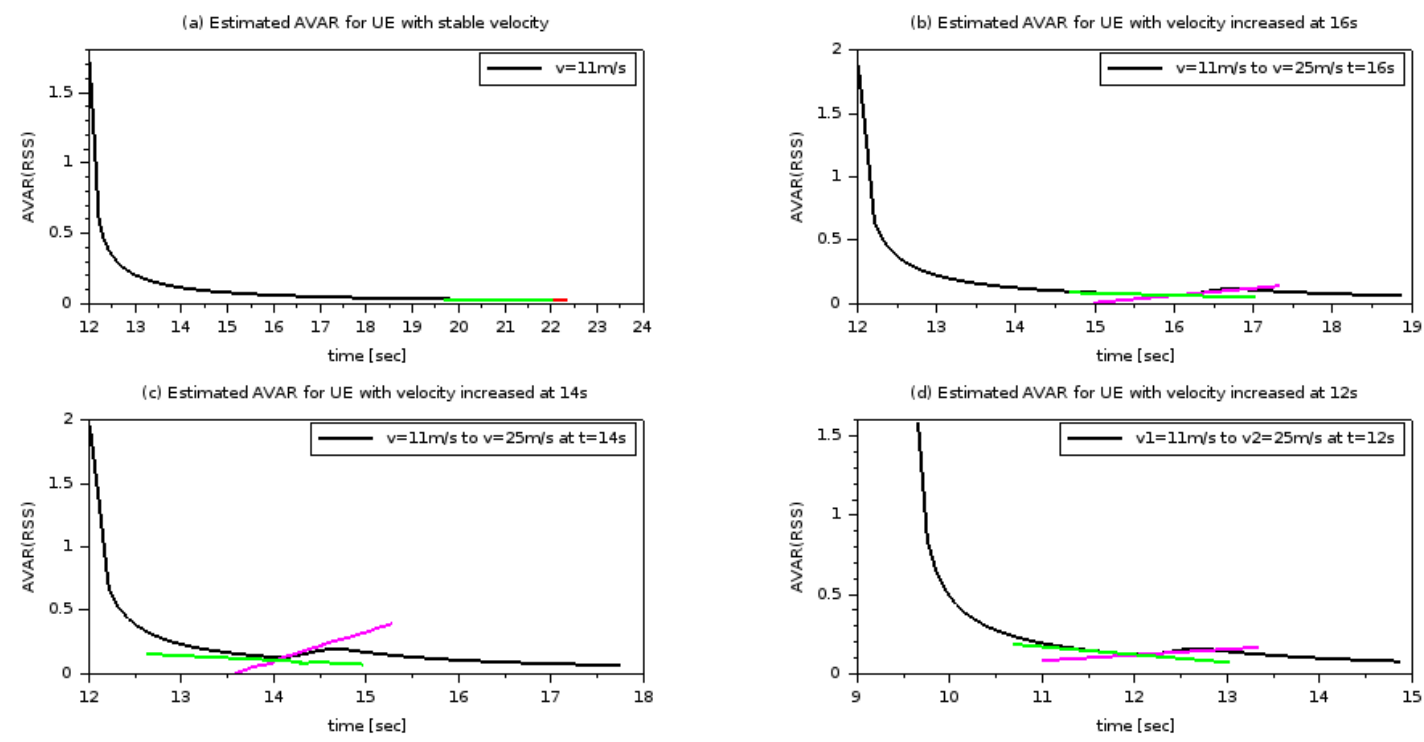

Figure 2. The Allan variance (AVAR) of the received signal strength (RSS) for the user equipment (UE) leaving the access point $(\mathrm{AP})$ area.

To study the case where the UE equipment approaches the source of the access point (AP) we chose to track values of AVAR for the received RSS by the serving AP where the UE changed its velocity from $11 \mathrm{~m} / \mathrm{s}$ to $25 \mathrm{~m} / \mathrm{s}$ at $t=8, t=9$, and $t=10$, as presented in Figure 2 . For the three cases, the $\Delta \Theta$ approves the condition $\Delta \Theta$ being higher than $1 \%$ at the same time when the UE accelerated. In contrast, in the case where the velocity remains constant, the value of $\Delta \Theta$ stays under $1 \%$. We repeated this for the UE moving a far distance from the AP, and in accordance this time the UE changed velocity from $11 \mathrm{~m} / \mathrm{s}$ to $25 \mathrm{~m} / \mathrm{s}$ at $t=12, t=14$, and $t=16$.
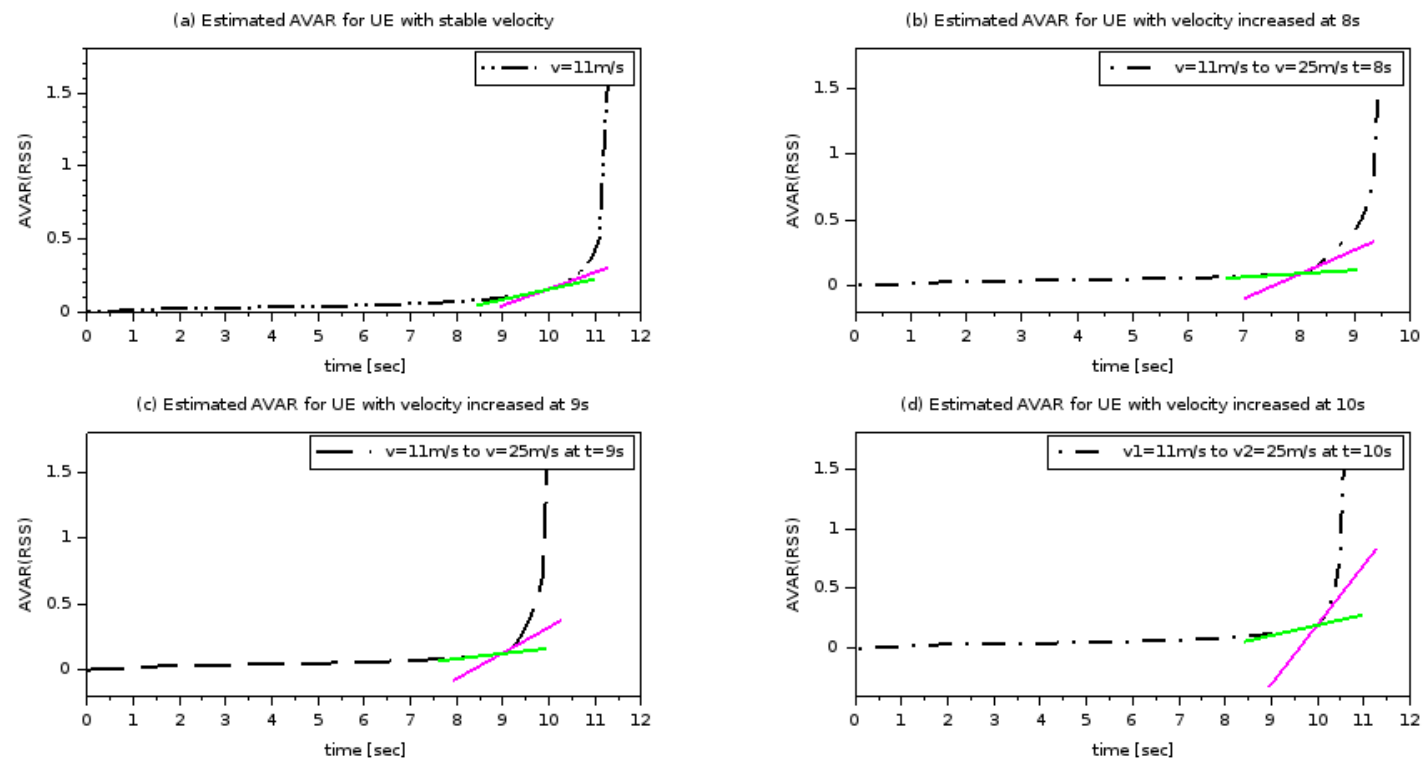

Figure 3. The AVAR of the RSS for the UE entering the AP area.

Figure 3 presents the behavior of tracked AVAR values, allowing us to make similar reflections as in Figure 2. Based on the experimental results, we found that making a decision based on $\Delta \Theta \geq 1 \%$ will help us to get the best result. This raises an interesting issue which is worth further investigation: What is the proper way to define the maximum value for $\Delta \Theta$ to target the process for handover? 


\subsection{System Architecture}

As a rule, the objective of handover is to pick the best service for clients, but preposterous parameters will influence the nature of the call. In this manner, we consolidated two systems, IEEE 802.21 MIH and PMIPv6, in order to perform a powerful handover. PMIPv6 is a network-based localized mobility management protocol that empowers IP mobility for the UE without involving its contribution to any mobility-related signaling. The components of the PMIPv6 standard are the Mobility Access Gateway (MAG) and the Local Mobility Anchor (LMA). The MAG performs mobility-related signaling, tracks the movement of the UE, and confirms the UE by trading restricting messages with the LMA. The LMA is a topological anchor point for the UEs that stores all the routing data and the UE's Home Network Prefix (HNP) to guarantee the UE's reachability state in the corresponding local domain [21]. Note that since the UE moves between various MAGs in PMIPv6, handover latency cannot be avoided. Along these lines, keeping in mind the end goal to diminish the handover latency, the UE needs to execute the MIH to empower the connection between cross-layers.

The MIH system is institutionalized by the IEEE MIH working group to maintain consistent mobility handover across heterogeneous wireless technologies. The MIH and PMIPv6 are used next to each other, keeping in mind the end goal of reducing handover latency and packet loss. This encourages the MIH to establish Media-Independent Handover Functions (MIHFs) to upgrade the handover execution to the clients with versatility crosswise over heterogeneous remote systems. The MIHF utilizes the gathered data from both UE and network infrastructure to give service abstraction to the upper layers utilizing an adequate interface type for the candidate network so it can enhance the experience of the UE by supporting handover in diverse technologies. This component helps the upper layer (for example, PMIPv6 and the MIH clients) to keep the service running and to allow service adjustments for various levels of QoS.

The nature of handover will be influenced by velocity, particularly when the velocity is high, which can cause a diverse range of handover-related failures (for example, too-late handover, too-early handover, and PP handover). However, the conventional technique does not track velocity. In these unique circumstances and for better decision-making, we will mull over the velocity and maintain the stability of handover. The entrance point occasionally sends guides from which we can remove RSS. Taking into account that the estimation of RSS is affected by the separation from the UE to AP, it is implicitly additionally impacted by velocity. This drives us to examine the variability of the RSS utilizing AVAR.

\subsection{Handover Process Description}

The primary concept of the recommended scheme is to foresee the handover choice, taking into account the velocity of the UE. Since the MIH can gather the RSS got from the serving AP, we utilize the MIHF functionality to obtain an N sample of RSS for $\Delta_{t}=\tau$.

In view of Equation (3), we calculate the variance $\sigma^{2}(\tau)$ continuously in order to track and check if $\Delta \Theta \geq 1 \%$. Once this condition is fulfilled, the UE will use the global positioning system (GPS) to get the velocity value. At the point when the velocity is accessible, the lifetime $\left((\Delta t)_{\text {lifetime }}\right)$ for the UE in the coverage area of the serving AP is recalculated utilizing the present velocity. Then, we confirm if $\left((\Delta t)_{\text {lifetime }}\right)$ is greater than the time required to play out a handover $\left((\Delta t)_{H O}\right)$, else we should begin the handover procedure to maintain a strategic distance from the HOF. Figure 4 resumes the process. To limit the PP impact, we ought to get the $\left((\Delta t)_{\text {lifetime }}\right)$ before becoming associated with the new AP. 


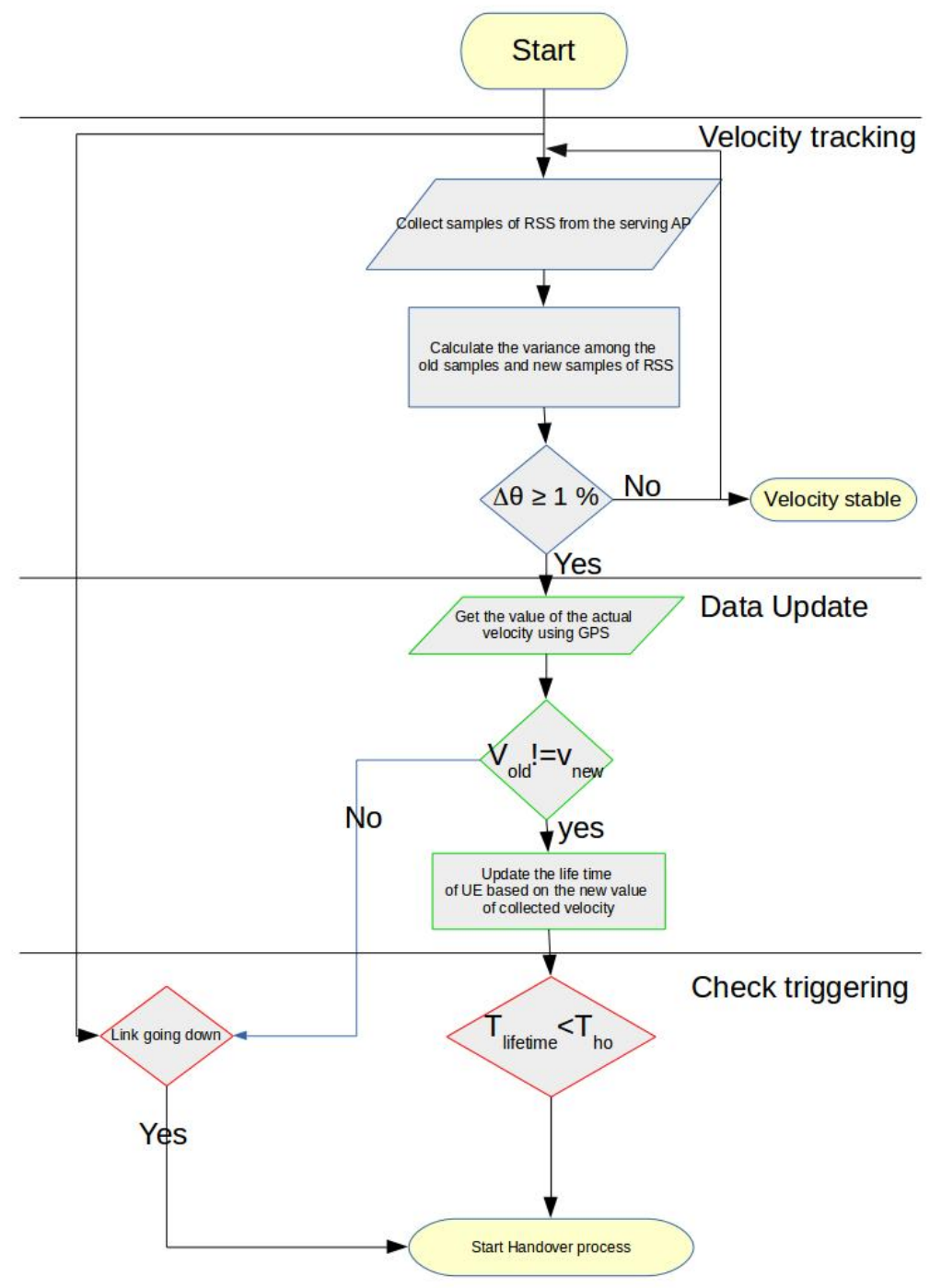

Figure 4. Flow chart for handover decision-based velocity.

In the wake of checking the condition $\left((\Delta t)_{\text {lifetime }}<(\Delta t)_{H O}\right)$, the UE sends the "link going down" primitive to trigger the handover procedure as demonstrated in Figure 5. The serving AP will begin gathering data from Media-Independent Information Services (MIISs) for seamless handover by sending "MIH get information" primitive. Once the data on neighboring APs is gathered, a serving $\mathrm{AP}$ starts to rank them in accordance with user preferences (in our situation the rating criterion reflect the value of the RSS). The consecutive stage will be the confirmation of resource availability in the candidate APs; this progression will be coordinated by the serving AP utilizing the "MIH N2N HO candidate query" primitive of the MIH system to limit the utilization of energy by the UE. At that point, the asset arrangement is overseen by the "MIH N2N HO commit" primitive. Now, we can perform PMIPv6 handover. Once the "link up" primitive is activated, at the new AP the PMIPv6 begins taking care of new node by sending a Proxy Binding Update (PBU) to the LMA. After the gathering of the PBU, the LMA refreshes the lifetime of the MN's entrance in the binding cache table and answers with a Proxy Binding Acknowledgment (PBA) message. At that point, begins transmission of the buffered data through the tunnel between the LMA and the new MAG. For resource discharge, the "MIH N2N HO complete" primitive is used. 


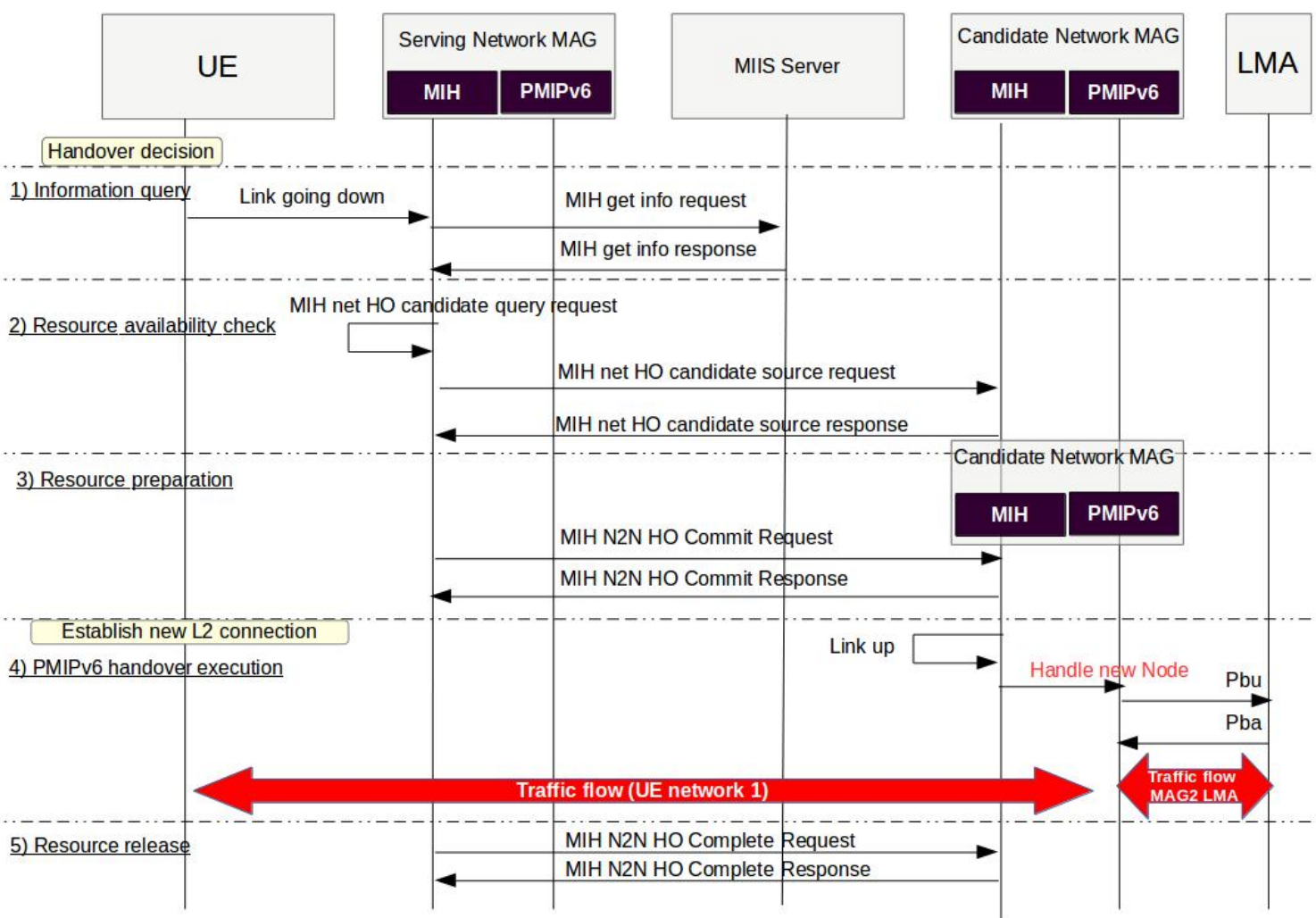

Figure 5. Operation procedure of the proposed IEEE 802.21 MIH functionality assisted by PMIPv6.

\section{Experiments and Analysis}

\subsection{Experimental Setup}

To manage the exponentially expanding number of data traffic requests, cell systems advance into heterogeneous networks (HetNets), where a substantial number of small cells (i.e., microcells, picocells, and femtocells) with broadly different transmit powers levels and physical sizes are randomly conveyed inside the coverage area of the macrocell [22]. The promising nature of HetNets has been broadly credited. Notwithstanding, using HetNets involves various difficulties, for example, mobility management.

The theoretical model created in the previous section is assessed here. The proposed scheme was executed in Network Simulator 3 (NS3). In the scenario, we created 30 mobile UE units, with only 21 UE units moving. We configured two different technologies, the LTE and WiFi network, by designing one EnbNode for full coverage, and six overlapping APs, as presented in Figure 6. For PMIPv6 protocol we used one LMA and three MAGs, where the UE moved between different MAGs.

Every UE unit is equipped with two interfaces enabling it to utilize the network appropriate to their needs. We assume that the UE is moving using the random walk scheme to an alternate MAG, and since the interval of medium velocity in urban and rural is (11-25 m/s), we will utilize those values for UE as irregular velocity. An uplink and downlink of user datagram protocol (UDP) traffic is transmitted amongst corresponding node (CN) and UE. The UDP packet size is equal to 100 bytes, while the time between successive packets was set at $1000 \mathrm{~s}$. For the two network technologies, we utilized the Range Propagation Loss Model, with a coverage area of LTE and WiFi that is equal to $500 \mathrm{~m}$ and $90 \mathrm{~m}$, respectively, and an overlapping area each two neighboring cells. 


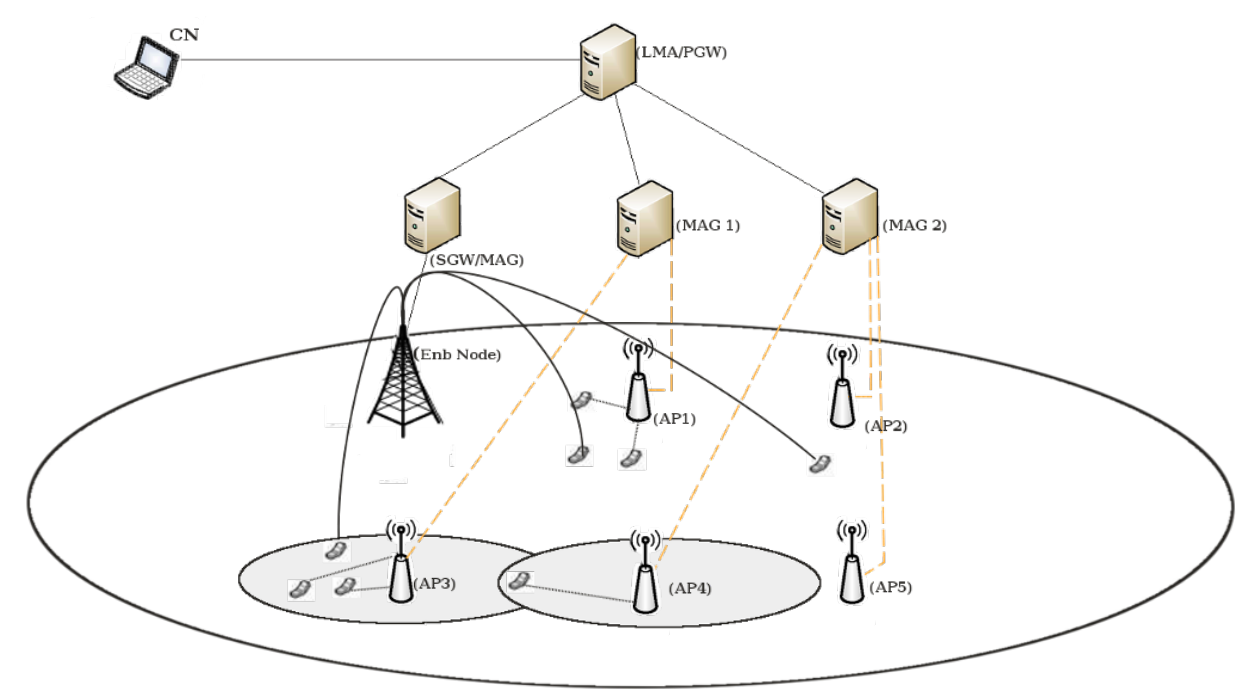

Figure 6. Heterogeneous network topology.

We set up a procedure that enables the mobile UE to increase or decrease its velocity while moving, in order to reproduce the issue in an urban situation. The simulation was repeated 30 times with the same movement features for each of the UE units. To enhance the exactness of the outcome, the presented results are the average value of these simulations results with a $95 \%$ confidence interval. The reenactment parameters are as delivered in Table 1.

Table 1. Simulation parameter values.

\begin{tabular}{ll}
\hline Parameters & Values \\
\hline Cell layout & 1 eNB, 6 APs \\
Propagation model & Range propagation loss model \\
LTE coverage & $500 \mathrm{~km}$ \\
WiFi coverage & $90 \mathrm{~m}$ \\
Mobility model & Random walk \\
Velocity & Urban $(11 \mathrm{~m} / \mathrm{s}, 25 \mathrm{~m} / \mathrm{s})$ \\
Packet size & 120 bytes \\
Number of UE units & 30 \\
Number of mobile UE units & 21 \\
Simulation time & $700 \mathrm{~s}$ \\
\hline
\end{tabular}

\subsection{Simulation Analysis}

The execution assessment of after effects of the proposed approach are dealt with using two distinct plans. The first is RSS MIH-PMIPv6, where handover decision-making is performed according to the RSS employing MIH-assisted-PMIPv6 for mobility administration. The second system, RSS-MIH, performs handover decision-making in view of the RSS supported by MIH for handover administration. The main features of the systems studied are exhibited in Table 2. This investigation of the framework achievement is expected to guarantee that the novel framework yields an improvement in handover performance.

Based on Figure 7, we can notice that at a moderate velocity, there is no extremely significant HOF rate. In any case, as the velocity grows, the HOF rate also grows. However, it grows in accordance with rate in the two other systems. This can be supported using the guidance of MIH standard for our proposed approach, which yields suitable tools for the sheltered use of the new proposed handover triggering technique. 
Table 2. Main features of the studied schemes.

\begin{tabular}{lccc}
\hline Main Characteristics & RSS-MIH & RSS-MIH-PMIPv6 & Proposed Scheme \\
\hline Supports handover in different technologies & $\checkmark$ & $\checkmark$ & $\checkmark$ \\
Considers the variation velocity & x & x & $\checkmark$ \\
Updates the lifetime of the UE & x & x & $\checkmark$ \\
Gives the UE the possibility of finishing handover properly & x & $\checkmark$ & $\checkmark$ \\
Manages environment data for decision-making & $\checkmark$ & $\checkmark$ & $\checkmark$ \\
Involves transparent mobility management for the UE & x & x & $\checkmark$ \\
Is a proactive and intelligent triggering system & x & &
\end{tabular}

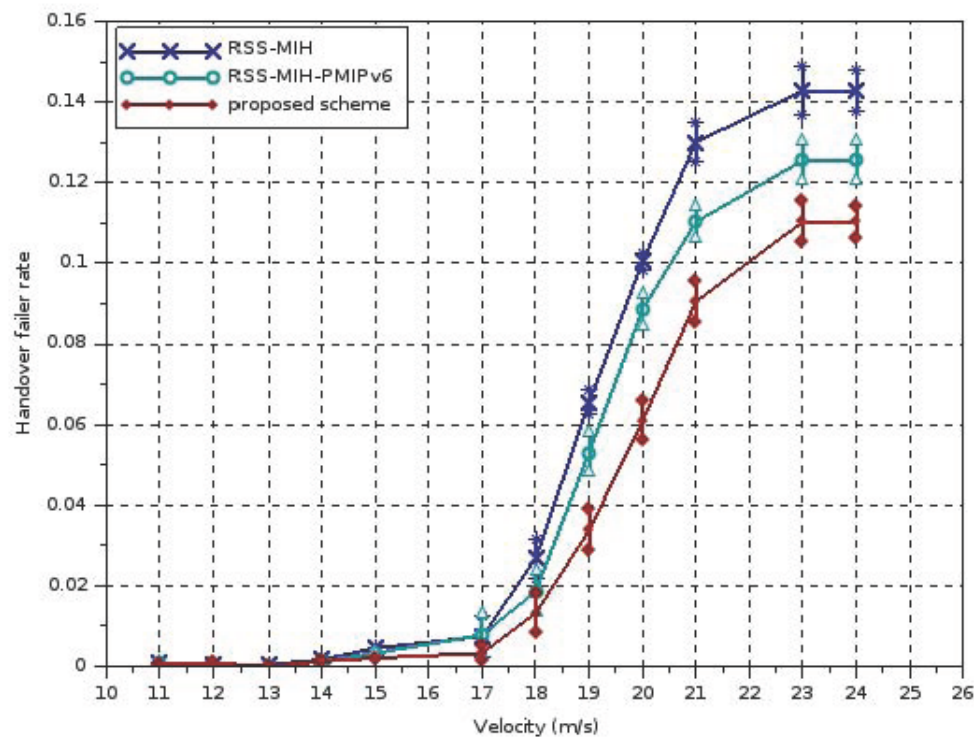

Figure 7. Handover failure (HOF) rate according to velocity. The confidence interval is $95 \%$.

Recognizing the AVAR of RSS values, the UE will check its velocity and then the $(\Delta t)_{\text {lifetime }}$ is revived. This framework causes the UE to prognosticate the handover procedure, which implies that UE will not be out of scope while the handover process is running. The delayed consequences of numerical examination show that a too-high movement rate of the UE will cause it to achieve a too-late handover process. Certainly, for a velocity equivalent to $24 \mathrm{~m} / \mathrm{s}$, the HOF rate can meet the request of $11 \%$, while this proportion increases to $12.5 \%$ and $14.5 \%$ with the RSS-MIH-PMIPv6 and RSS-MIH plots, respectively. This can be interpreted by the UE moving in the cell edges; since the UE's movement is unpredictable, we can resolve this in an urban situation using an ideal organization of AP location.

Table 3 shows the framework asset usage rate for the LTE and the WiFi networks. The rate of asset use is examined with respect to the UE velocity. In fact, an expansion in velocity pushes the mobile UE to pick the given LTE services. The UE rapidly leaves the Wireless Local Area Network (WLAN) network, which is defined by a restricted radio coverage, as opposed to the LTE network.

Table 3. Mobile user equipment (UE) behavior in HetNets according to velocity.

\begin{tabular}{lllllllllllll}
\hline Velocity (m/s) & $\mathbf{1 1}$ & $\mathbf{1 2}$ & $\mathbf{1 3}$ & $\mathbf{1 4}$ & $\mathbf{1 5}$ & $\mathbf{1 7}$ & $\mathbf{1 8}$ & $\mathbf{1 9}$ & $\mathbf{2 0}$ & $\mathbf{2 1}$ & $\mathbf{2 3}$ & $\mathbf{2 4}$ \\
\hline LTE & $19 \%$ & $20 \%$ & $21 \%$ & $25 \%$ & $29 \%$ & $30 \%$ & $39 \%$ & $42 \%$ & $49 \%$ & $50 \%$ & $65 \%$ & $70 \%$ \\
WiFi & $81 \%$ & $80 \%$ & $79 \%$ & $75 \%$ & $71 \%$ & $70 \%$ & $61 \%$ & $58 \%$ & $51 \%$ & $50 \%$ & $35 \%$ & $30 \%$ \\
\hline
\end{tabular}

Such outcomes can be judged as eminent outcomes and reflect a refinement in radio asset performance that keeps up with the simulation reproduction. The reason behind this improved 
utilization of the assets is because of the rationality of our handover decision-making, which makes it conceivable to modify the handover trigger quickly.

Handover failure has a fundamental role in the discontinuance of services, and it affects the packet loss rate. Since the handover is not achieved successfully, the association with another network consequently requires increased time. The packet loss rate, which denotes the ratio of packet loss value and the total number of sent packets, will be evaluated and presented over velocity in Figure 8. This demonstrates that our proposed system beats the RSS-MIH-PMIPv6 and RSS-MIH systems.

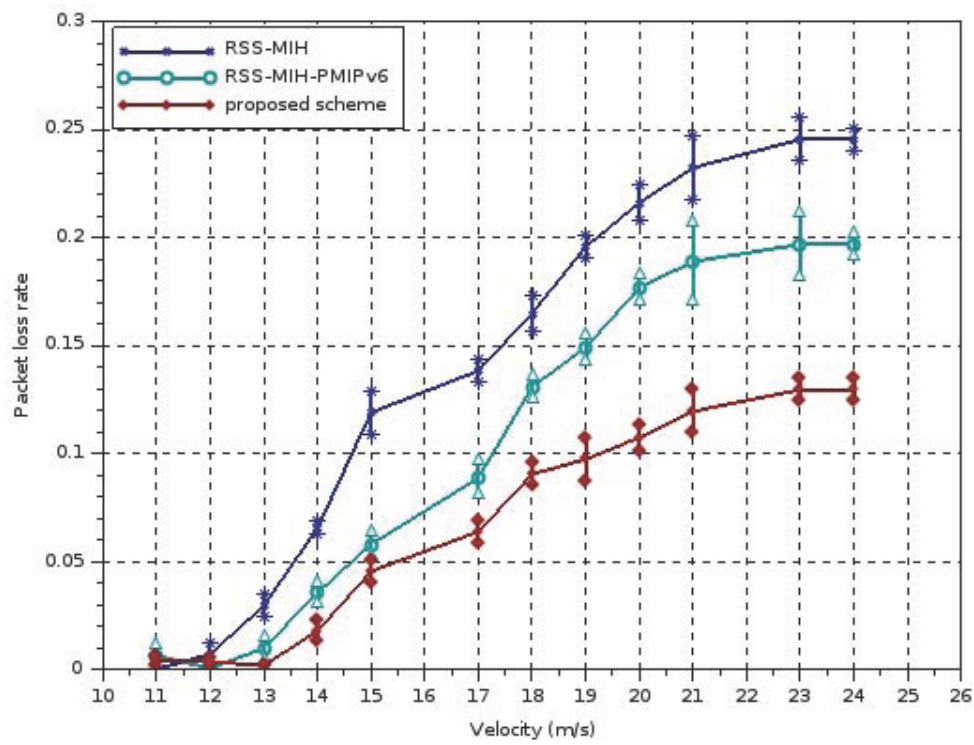

Figure 8. Packet loss rate according to velocity. The confidence interval is $95 \%$.

We can presume that the development of packet loss rate begins from a velocity corresponding to $15 \mathrm{~m} / \mathrm{s}$, and the rate starts to develop and achieves an estimation of $10 \%$ for a velocity equivalent to $19 \mathrm{~m} / \mathrm{s}$. However, for the other system, the average rate of packet loss can reach $20 \%$. The motivation behind why the packet loss rate of the proposed scheme is consistently lower than in the other systems is because of the intervention of the anticipating handover triggering technique, which allows the UE to make a decision before degradation of the QoS.

From that point forward, for the proposed scheme the packet loss rate keeps on expanding similarly until reaching $12.5 \%$ and $13 \%$ for velocities equivalent to $23 \mathrm{~m} / \mathrm{s}$ and $24 \mathrm{~m} / \mathrm{s}$, respectively, where it begins to increment in a passive manner. For other schemes, these rates increase to $19 \%$ and $25 \%$, respectively.

The delay includes the full period of execution of the process; this period begins at the time of sending a packet from the source, continuing until it is admitted by the destination. For our situation, handover delay is calculated from the time the UE sends a link-going-down message to begin the handover procedure, until the connection with the new serving BS is built. We will concentrate on the changes brought about by PMIPv6 to MIH for handover delay.

Figure 9 trick the normal handover delay of the UE moving at various speeds. It demonstrates that our proposed policy in RSS-MIH-PMIPv6 scheme outperforms the RSS-MIH scheme, and shows that handover delay diminishes proportionately as UE velocity grows. As an interpretation, we note that the quicker the UE travels, the shorter the time expected to surpass the distance in order to recognize the occurrence of new competitor BS. This helps to lessen the time-scanning interval. In addition, according to Table 3, at a velocity higher than $21 \mathrm{~m} / \mathrm{s}$ the favored populace is the LTE network, in which the delay was enhanced by $0.15 \mathrm{~ms}$ since the lifetime condition encouraged the client to keep LTE as the only option. Along these lines, access to LTE is made specifically without passing through the resource availability stage. 


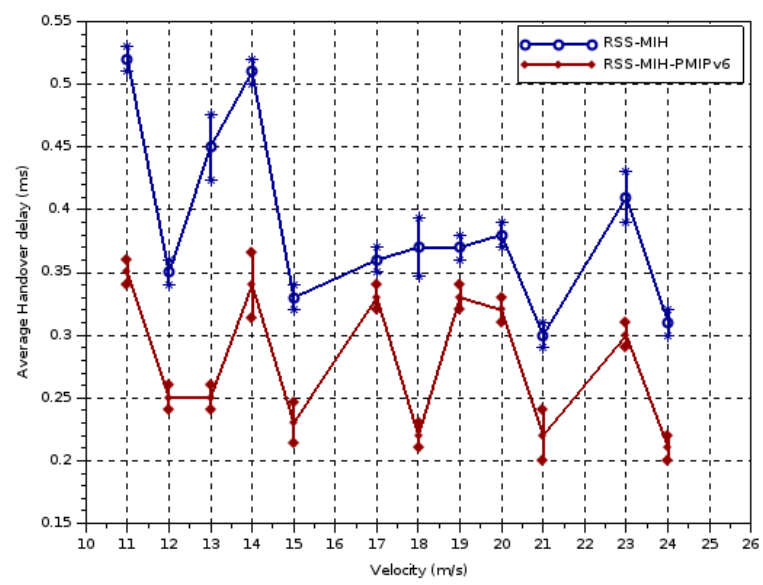

Figure 9. Handover delay according to velocity. The confidence interval is $95 \%$.

\section{Discussion and Future Directions}

In this article, we inaugurated a general handover decision-making framework which can be bolstered by future system models. An astute decision-making technique and workflow procedure for the heterogeneous wireless network was presented in this paper. An extensive examination of our approach and other conventional plans is shown in Section 4.

Our approach is general and can be bolstered by future system structures (for example LTE Pro, 5G, cell Internet of Things (IoT), and so-forth). The objectives of such handover decision-making are to diminish HOFs, lessen the packet loss, meet the QoS prerequisite for the velocity of all UE units, and preserve limited and useful signaling flow. Each of these objectives has its difficulties to be addressed in this new network approach.

A workflow of the suggested MIH-assisted PMIPv6 for the heterogeneous network system is exhibited in Figure 5. The proposed triggering scheme consists of three levels. The initial level incorporates MIH to empower the UE with numerous interfaces to work within the 3GPP LTE and 5G WiFi interfaces. MIH grants UEs with bolstered mobility in a heterogeneous network, and makes the services outfitted by the MIHF more appropriate in order to guarantee decent inter-layer communication for consistent handover, as required by mobile UE. The second level involves updating the crucial parameter values, velocity, and lifetime for decision-making in the most relevant situations (not too late, and not too early). Ultimately, the arrangement of triggering is restored and for decent reproduction, our approach introduces two triggering conditions: signal degradation or the lifetime, converging towards the handover time execution. If one of the conditions is legitimate, an official announcement of the handover is made. The MIIS, which allows the UE to collect data on the heterogeneous network existing in a geographical area, has been executed in the core network. The objective of arrangement is to have a total perspective of the system by providing full fundamental data on the access network to make strong decisions for mobility management and to perform different activities.

Soon, we intend to coordinate our examination towards 5G heterogeneous network systems by adjusting and reformulating the way we deal with traffic models for Cellular IoT, as described in 3GPP release 13 [23]. It is important to examine new arrangements, for example, location-aware handover, and research how to profit from Network Function Virtualization, Software-Defined Networking, and cloud service technologies for mobility management systems of mobile network services. In future work we will examine new ideas associated with the management of mobility, for example resource virtualization and resource abstraction. 


\section{Conclusions}

We built up a situation of correspondence in a two-tier heterogeneous network with one primary motivation: to anticipate the decision-making of handover before UE remoteness from the serving AP caused the degradation of the QoS. Utilizing MIH framework gives the user the possibility to gather pertinent data that will assume a critical part in triggering the handover process. With respect to this information, we are concerned with RSS, which is wrapped in the beacons sent occasionally by the AP. To apply the concept of velocity supervision, we use the RSS AVAR, bearing in mind the ultimate goal of identifying the change in velocity. Thus, we recalculate $(\Delta t)_{\text {lifetime }}$ for the present velocity. Once the choice is made, MIH passes the handover to PMIPv6, which is then in charge of administering the mobile IP functionality. Simulation results demonstrated that the amount of handover failure and packet loss can be diminished.

Author Contributions: Asmae Ait Mansour: main writing, design of the overall system, analysis, and improvement of the proposed system; Nourddine Enneya and Mohamed Ouadou: overall supervision of the paperwork, review, and comments, etc. All authors have read and approved the final manuscript.

Conflicts of Interest: Authors declare no conflict of interest.

\section{References}

1. Lai, W.K.; Chiu, J.C. Improving handoff performance in wireless overlay networks by switching between two-layer ipv6 and one-layer ipv6 addressing. IEEE J. Sel. Areas Commun. 2005, 23, 2129-2137.

2. Mahbas; Ali; Zhu, H.; Wang, J. Mobility Management in Small Cell Networks. In Proceedings of the IEEE Globecom 2017, Singapore, 4-8 December 2017.

3. Zhou, Y.; Ai, B. Handover schemes and algorithms of high-speed mobile environment: A survey. Comput. Commun. 2014, 47, 1-15.

4. Mansour, A.A.; Enneya, N.; Ouadou, M. A Seamless Handover Based MIH-Assisted PMIPv6 in Heterogeneous Network (LTE-WIFI). In Proceedings of the 2nd International Conference on Big Data, Cloud and Applications, Tetouan, Morocco, 29-30 March 2017.

5. Baddour, K.E.; Beaulieu, N.C. Robust doppler spread estimation in nonisotropic fading channels. IEEE Trans. Wirel. Commun. 2005, 4, 2677-2682.

6. Arvind, M.; Güvenc, I. Handover count based velocity estimation and mobility state detection in dense HetNets. IEEE Trans. Wirel. Commun. 2016, 15, 4673-4688.

7. Merwaday, A.; Güvenç, I.; Saad, W.; Mehbodniya, A.; Adachi, F. Sojourn time-based velocity estimation in small cell poisson networks. IEEE Commun. Lett. 2016, 20, 340-343.

8. Merwaday, A.; Ismail, G. Specific Velocity Estimation of Wireless Network Devices. U.S. Patent 9,584,976, 28 February 2017.

9. Arshad, R.; ElSawy, H.; Sorour, S.; Al-Naffouri, T.Y.; Alouini, M.-S. Velocity-aware handover management in two-tier cellular networks. IEEE Trans. Wirel. Commun. 2017, 16, 1851-1867.

10. Zhang, R.; Wu, M.; Zhang, Y. Analysis of handover trigger scheme based on distance for LTE high-speed railway networks. TELKOMNIKA Telecommun. Comput. Electron. Control 2016, 14, 129-135.

11. Yang, Y.; Yu, P.; Li, W. Handover self-optimization mechanism based on velocity for cellular networks. In Proceedings of the 2012 7th International ICST Conference on Communications and Networking in China (CHINACOM), Kunming, China, 8-12 August 2012; pp. 606-610.

12. Xu, P.; Fang, X.; He, R.; Xiang, Z. An efficient handoff algorithm based on received signal strength and wireless transmission loss in hierarchical cell networks. Telecommun. Syst. 2013, 52, 317-325.

13. Jain, A.; Tokekar, S. Optimization of vertical handoff in UMTS_WLAN heterogeneous networks. In Proceedings of the International Conference on Emerging Trends in Communication, Control, Signal Processing \& Computing Applications, Bangalore, India, 10-11 October 2013; pp. 1-5.

14. Sinclair, N.; Harle, D.; Glover, I.A.; Irvine, J.; Atkinson, R.C. An advanced som algorithm applied to handover management within lte. IEEE Trans. Veh. Technol. 2013, 62, 1883-1894.

15. Park, J.; Lim, Y. A handover prediction model and its application to link layer triggers for fast handover. Wirel. Pers. Commun. 2010, 52, 501-516. 
16. Roy, A.; Shin, J.; Saxena, N. Multi-objective handover in lte macro/femto-cell networks. J. Commun. Netw. 2012, 14, 578-587.

17. Shi, J.; Wu, J.; Paul, A.; Jiao, L.; Gong, M. Change detection in synthetic aperture radar images based on fuzzy active contour models and genetic algorithms. Math. Probl. Eng. 2014, doi:10.1155/2014/870936.

18. Luo, C.; Higuera, P.C.; McClean, S.; Parr, G.; Grecos, C. Analysis of coloured noise in received signal strength using the allan variance. In Proceedings of the 2014 22nd European Signal Processing Conference (EUSIPCO), Lisbon, Portugal, 1-5 September 2014; pp. 994-998.

19. IEEE Standard, Definitions of Physical Quantities for Fundamental Frequency and Time Metrology-Random Instabilities; IEEE Standard 1139-2008; IEEE: Piscataway Township, NJ, USA, 2009.

20. Lesage, P.; Ayi, T. Characterization of frequency stability: Analysis of the modified allan variance and properties of its estimate. IEEE Trans. Instrum. Meas. 1984, 33, 332-336.

21. Kong, K.-S.; Lee, W.; Han, Y.-H.; Shin, M.-K.; You, H. Mobility management for all-ip mobile networks: Mobile ipv6 vs. proxy mobile ipv6. IEEE Wirel. Commun. 2008, 15, 36-45.

22. Andrews, J.G.; Buzzi, S.; Choi, W.; Hanly, S.V.; Lozano, A.; Soong, A.C.K.; Zhang, J.C. What will $5 \mathrm{~g}$ be? IEEE J. Sel. Areas Commun. 2014, 32, 1065-1082.

23. 3GPP. Study on Architecture Enhancements for Cellular Internet of Things (Release 13); 3rd Generation Partnership Project TR 23.720; 3GPP: Phoenix, AZ, USA, 2016.

(C) 2018 by the authors. Licensee MDPI, Basel, Switzerland. This article is an open access article distributed under the terms and conditions of the Creative Commons Attribution (CC BY) license (http://creativecommons.org/licenses/by/4.0/). 